\title{
Analytical representation of nonlinear Froude-Krylov forces for 3-DoF point absorbing wave energy devices
}

\author{
Giuseppe Giorgi*, John V. Ringwood \\ Centre for Ocean Energy Research, Maynooth University, Maynooth, Co. Kildare, Ireland
}

\section{A R T I C L E I N F O}

\section{Keywords:}

Nonlinear Froude-Krylov forces

Wave energy converters

Multi degrees of freedom

Pitching instability

Computational efficiency

Control optimization

\begin{abstract}
A B S T R A C T
Accurate and computationally efficient mathematical models are fundamental for designing, optimizing, and controlling wave energy converters. Many wave energy devices exhibit significant nonlinear behaviour over their full operational envelope, so nonlinear models may become indispensable.

Froude-Krylov nonlinearities are of great importance in point absorbers but, in general, their calculation requires an often unacceptable increase in model complexity/computational time. However, for axisymmetric bodies, it is possible to describe the whole geometry analytically, thereby allowing faster calculation of nonlinear Froude-Krylov forces.

In this paper, a convenient parametrization of axisymmetric body geometries is proposed, applicable to devices moving in surge, heave, and pitch. While, in general, Froude-Krylov integrals must be solved numerically, by assuming small pitch angles, it is possible to simplify the problem, and achieve a considerably faster algebraic solution. However, both nonlinear models compute in real-time.

The framework presented in the paper offers flexibility in terms of computational and fidelity levels, while still representing important nonlinear phenomena such as parametric pitch instability. Models with lower computational requirements may be more suitable for repetitive calculations, such as real-time control, or longterm power production assessment, while higher fidelity models may be more appropriate for maximum load estimation, or short-term power production capability assessment.
\end{abstract}

\section{Introduction}

Mathematical models are indispensable for designing, optimizing, and controlling wave energy converters (WECs). Ideally, such models are required to be both accurate and computationally efficient. The most popular models are linear, which are convenient for their short computation time, but accurate only for small relative fluid/body motions. Conversely, wave energy converters are likely to experience large movements, especially under controlled conditions, in order to maximize the power absorption. Consequently, significant nonlinear effects may arise, so that linear models become less reliable (Giorgi and Ringwood, 2017c).

The inclusion of nonlinear terms in the equation of motion generally improves the accuracy of the model, but with additional complexity and computational burden. In particular, it has been shown, in the literature, that nonlinear Froude-Krylov (FK) forces, which represent the integral of the static and dynamic pressure over the wetted surface of the device, are especially important for point absorbers (Giorgi and Ringwood, 2017a). Furthermore, nonlinear FK forces are responsible for purely-nonlinear phenomena, such as pitching instability or parametric roll (Tarrant, 2015).

For geometries of arbitrary complexity, the computation of nonlinear FK forces first requires the discretization of the surface with a mesh, and then the employment of a time-consuming remeshing routine, at each time step, in order to calculate the instantaneous wetted surface of the device (Matusiak, 2011; Bandyk, 2009). However, if the body is assumed to be axisymmetric, it is possible to describe the complete geometry analytically, thereby avoiding the use of a mesh (Giorgi and Ringwood, 2017b). Note that such a hypothesis is not particularly restrictive, since the vast majority, if not all, point absorbers are designed to be non-directional, and are therefore axisymmetric. Due to the analytical description of the geometry, the computation of nonlinear FK forces is considerably faster than the meshing approach.

In this paper, a convenient parametrization of axisymmetric surfaces is proposed, applicable to devices moving in three degrees of freedom (DoFs), allowing an analytical description of nonlinear FK forces in surge, heave, and pitch. In general, the FK integrals must be solved numerically using, for example, a trapezoidal rule. Assuming

\footnotetext{
* Corresponding author.

E-mail address: giuseppe.giorgi.2015@mumail.ie (G. Giorgi).
} 
small pitch angles, however, it is possible to further simplify the problem, and achieve an algebraic solution, which is considerably faster than numerical integration. Hereafter, LFK is used as the acronym for linear FK forces, VFK for the algebraic-nonlinear model (since it assumes a vertical geometry), and RFK for the numerical-nonlinear model (since it considers a rotating geometry).

The application of these three different approaches to the FK force calculation (linear, algebraic-nonlinear, and numerical-nonlinear) may depend on the particular purpose the mathematical model is intended for. For preliminary studies, shape optimization, or WEC farm configuration analysis, many iterations are required; therefore, the requirement for fast calculation prevails over the accuracy requirement. In case of control optimization routines, a higher level of accuracy is of great importance, at a significantly low computational time; therefore, the algebraic-nonlinear approach may be the most appropriate. Finally, higher degree of accuracy is needed for power production assessment, or to compute maximum loads (for the design of the mechanical properties of the structure and mooring lines), or for verifying the likelihood of events such as instability or parametric roll. In such cases, the numerical-nonlinear method may be preferred.

However, the choice between the LFK, VFK, and RFK, strongly depends on the operational space spanned by the device in its operating conditions, in particular the heave displacement and the pitch angle. In fact, for small motions, linear assumptions are reasonably valid, and all models effectively overlap. Conversely, when the device experiences large motions, typically induced by the control strategy, important differences between the models may arise.

The purpose of this paper is to provide a simple and computationally convenient formulation for nonlinear FK forces for axisymmetric wave energy converters, moving in surge, heave, and pitch. A case study is then considered, inspired by the CorPower device (CorPower, 2017), in order to quantify differences in accuracy and computation time for linear, algebraic-nonlinear and numerical-nonlinear models. Previous studies, in the literature, concerning nonlinear FK forces for multi-DoF wave energy devices, use a computationally expensive meshbased approach (Penalba et al., 2017), while this paper introduces a more efficient methodology, applicable to point absorbing WECs.

Although such a modelling approach for nonlinear FK forces calculations was already proposed in (Giorgi and Ringwood, 2017b), only very academic case studies were considered, such as spheres, while this paper demonstrates the applicability of the method to a real device; this allows a realistic quantification of the computational efforts related to a geometry composed of different elementary geometries, as well as the discussion of more realistic nonlinear effects. A further novelty of this paper is the expansion of the method to multiple DoFs, as opposed to 1DoF as in (Giorgi and Ringwood, 2017b). In fact, two solutions are proposed and discussed (VFK or RFK), with significantly different computational burdens (about two orders of magnitude difference). The choice between algebraic (VFK) or numerical (RFK) integrations is guided by the accuracy/computational compromise, specific to the particular application the model is intended to serve. Furthermore, the expansion to 3-DoFs is not trivial, especially for the numerical integration solution (RFK): some practical issues are here addressed, leading to two different approaches, one of which is almost twice as fast as the other.

The reminder of the paper is organized as follows: Sect. 2 presents the different methods to compute FK forces, which are validated in Sect. 3. A parametric study is proposed in Sect. 4, while the dynamical response to incoming waves is discussed in Sect. 5. Some final remarks and conclusions are given in 6 . An appendix is included as well, in order to explicitly provide all the algebraic results, obtained with the VFK model.

\section{Froude-Krylov forces}

In the framework of linear potential theory, FK forces correspond to the integral of the pressure of the undisturbed wave field over the wetted surface of the device. Such a pressure is defined, according to linear Airy's theory, as:

$p(x, z, t)=p_{s t}+p_{d y}=-\gamma z+\gamma a \frac{\cosh (\chi(z+h))}{\cosh (\chi h)} \cos (\omega t-\chi x+\varphi)$

where $p_{s t}=-\gamma z$ is the static pressure, $p_{d y}$ the dynamic pressure, $\gamma$ the specific weight of the sea water, $a$ the wave amplitude, $\chi$ the wave number, $\omega$ the wave frequency, $\varphi$ an arbitrary phase (usually set to zero), $h$ the water depth (defined according to a right-handed inertial frame of reference $(x, y, z)$, with the origin at the still water level (SWL)), $x$ pointing in the direction of propagation of the wave, and $z$ pointing upwards. The free surface elevation $\eta$ is defined as

$\eta(x, t)=a \cos (\omega t-\chi x+\varphi)$

Froude-Krylov forces are computed by integrating the pressure, shown in equation (1), over the instantaneous wetted surface $S(t)$. In particular, static and dynamic FK force components can be defined, respectively, as follows:

$\mathbf{F}_{F K_{S t}}=\mathbf{F}_{g}+\iint_{S(t)}-\gamma z \mathbf{n} d S$

$\mathbf{F}_{F K_{d y}}=\iint_{S(t)} p_{d y} \mathbf{n} d S$

where $\mathbf{n}=\left(n_{x}, n_{y}, n_{z}\right)$ is the unit vector normal to the surface, pointing outwards, and $\mathbf{F}_{g}$ is the gravity force. Likewise, FK torques are defined as follows:

$\mathbf{T}_{F K_{s t}}=\mathbf{r} \times \mathbf{F}_{g}+\iint_{S(t)}-\gamma z \mathbf{r} \times \mathbf{n} d S$

$\mathbf{T}_{F K_{d y}}=\iint_{S(t)} p_{d y} \mathbf{r} \times \mathbf{n} d S$

where $\mathbf{r}$ is the position vector, and $\times$ is the cross product.

For a geometry of arbitrary complexity, it is not possible to solve the FK integrals of (3a) to (4b). Linear boundary-element solvers linearize the problem around the still water level (SWL: $z=\eta=0$ ), therefore considering a constant wetted surface, unlikely to be valid for a WEC under energy-maximizing control (Giorgi and Ringwood, 2017c).

Alternatively, the geometry can be discretized through a mesh, computing the contribution to the force over each mesh panel (Gilloteaux, 2007). Such an approach, though feasible, is computationally expensive, due to the recalculation, at each time step, of the instantaneous wetted surface, and consequent remeshing of the geometry. For axisymmetric buoys, a convenient parametrization of the wetted surface can ease the calculation of the FK integrals. In particular, computationally efficient algebraic solutions of the FK integrals exist for vertical axisymmetric buoys (Giorgi and Ringwood, 2017b). Such a method is further described in Sect. 2.1.

If the body is also pitching, numerical integration is required. Such a method is further described in Sect. 2.2. Table 1 summarises the main different characteristics of LFK, VFK, and RFK, highlighting different assumptions and, qualitatively, different computational time requirements. Quantitative accuracy and computational time comparisons are presented in Sects. from 3 to 5 .

\subsection{Nonlinear Froude-Krylov force: algebraic integration}

Both the algebraic (VFK) and the numerical (RFK) integration approaches rely on the assumption of axisymmetric geometry, which allows the analytical description of the whole wetted surface. The geometry of a generic buoy which is symmetric around a vertical axis can be described in cylindrical coordinates, as follows:

$\left\{\begin{array}{l}x(\rho, \theta)=x_{G}+f(\rho) \cos \theta \\ y(\rho, \theta)=f(\rho) \sin \theta \\ z(\rho, \theta)=z_{G}+\rho\end{array}, \quad \theta \in[-\pi, \pi) \wedge \rho \in\left[\rho_{1}, \rho_{2}\right]\right.$ 
Table 1

Summary of the main difference between the three Froude-Krylov modelling approaches for axisymmetric buoys.

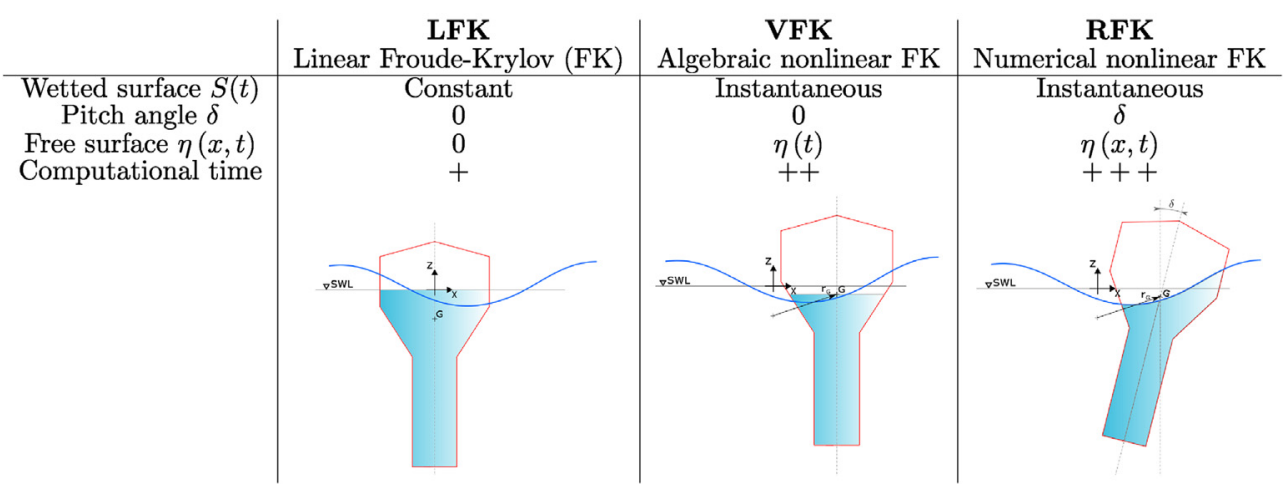

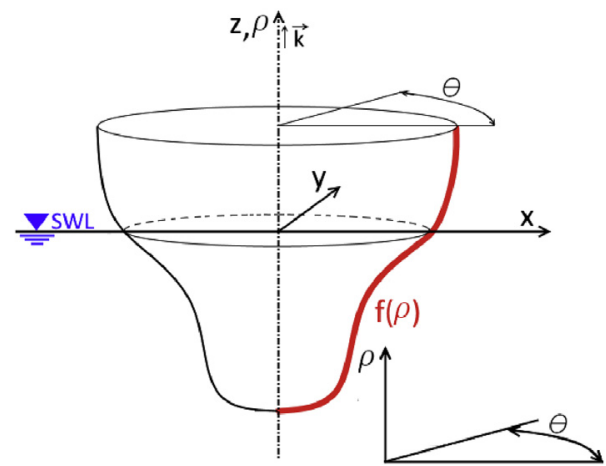

Fig. 1. Axisymmetric vertical device with generic profile of revolution $f(\rho)$.

where $f(\rho)$ is a generic function of the vertical coordinate $\rho$, describing the profile of revolution of the axisymmetric body, as shown in Fig. 1. The centre of gravity $(\mathrm{CoG})$ which, at rest, is assumed to lie on the axis at $\left(0,0, z_{C O G}\right)$, and moves about the $x-$ and $z-$ axis of $x_{G}$ and $z_{G}$, respectively.

The change of coordinates, from Cartesian $(x, y, z)$ to cylindrical $(\rho, \theta)$, requires the inclusion of $\left\|\mathbf{e}_{\rho} \times \mathbf{e}_{\theta}\right\|$ in the integral, where $\mathbf{e}_{\rho}$ and $\mathbf{e}_{\theta}$ are unity vectors in the $\rho$ and $\theta$ directions, respectively. Furthermore, $\mathbf{n}$ can be expressed as $\frac{\mathbf{e}_{\rho} \times \mathbf{e}_{\theta}}{\left\|\mathbf{e}_{\rho} \times \mathbf{e}_{\theta}\right\|}$. Simplifying the denominator of $\mathbf{n}$, it follows that the integral in ( $3 \mathrm{~b})$, for example, becomes:

$\mathbf{F}_{F K_{d y}}=\iint_{S(t)} p_{d y}(x, y, z) \mathbf{n} d S=\int_{\theta_{1}}^{\theta_{2}} \int_{\rho_{1}}^{\rho_{2}} p_{d y}(\rho, \theta)\left(\mathbf{e}_{\rho} \times \mathbf{e}_{\theta}\right) d \rho d \theta$

The cross product of the unity vectors is defined as follows:

$\mathbf{e}_{\rho} \times \mathbf{e}_{\theta}=\left(\begin{array}{c}f^{\prime}(\rho) \cos \theta \\ f^{\prime}(\rho) \sin \theta \\ 1\end{array}\right) \times\left(\begin{array}{c}-f(\rho) \sin \theta \\ f(\rho) \cos \theta \\ 0\end{array}\right)=f(\rho)\left(\begin{array}{c}-\cos \theta \\ -\sin \theta \\ f^{\prime}(\rho)\end{array}\right)$

Likewise, for the torque integrals:

$\mathbf{r} \times\left(\mathbf{e}_{\rho} \times \mathbf{e}_{\theta}\right)=\left(\begin{array}{c}f(\rho) \cos \theta \\ f(\rho) \sin \theta \\ \rho\end{array}\right) \times f(\rho)\left(\begin{array}{c}-\cos \theta \\ -\sin \theta \\ f^{\prime}(\rho)\end{array}\right)$

Integrals, such as (6), can be algebraically solved, for a vertical axisymmetric buoy, only if $\rho_{2}(\theta)$ (the top limit of integration) is horizontal. Therefore, $\rho_{2}(\theta)$ is set constant, and equal to the free surface elevation at the axis of the buoy $(\bar{\eta})$. Such a condition is relatively accurate if the wave length is much longer than the characteristic horizontal dimension of the device at the free surface elevation, which is often the case. Although $\rho_{2}$ is constant, the pressure field does change along the $x$ direction. In order to permit an algebraic solution, the cosine term of the pressure formulation, shown in (1), must be replaced with its McLaurin expansion, introducing a small error, as discussed in (Giorgi and Ringwood, 2017b).

One further simplification, needed to achieve an algebraic solution, is to consider the pressure formulation in infinite water-depth. The approximation introduced is normally negligible, given that point absorbers are usually installed in deep-water locations. Finally, note that, for the computation of nonlinear FK forces, it is advantageous to apply Wheeler stretching to the dynamic pressure in equation (1) (Giorgi and Ringwood, 2018b).

In Appendix A, the algebraic solution to the nonlinear FK force integrals is provided, considering two common geometries (cylinders, cones). In fact, the vast majority of axisymmetric point absorbers can be described as a combination of cylinders and cones. However, the discs (lids), which close the surface of a cylinder, cannot be described using cylindrical coordinates. To this end, polar coordinates are used to the describe any closing disc. Polar coordinates and algebraic solutions for a disc are provided in Appendix A.2.

\subsection{Nonlinear Froude-Krylov forces: numerical integration}

Calculating forces for a rotating body requires consideration of the coordinate system in which such forces are going to be represented. In particular, the dynamical equations may be written in the inertial global frame, or in the non-inertial body-fixed frame. In order to have a constant and minimal inertial matrix, it is usually convenient to employ a frame of reference fixed on the body, and with its origin at the body centre of gravity. Therefore, FK forces, in the body-frame, can be equivalently represented in two ways:

1. Formulating and solving the integral in the global frame, then transferring the results to the body-frame.

2. Formulating and solving the integral directly in the body-frame.

Option (a) requires the definition of the geometry in the global frame, which can be achieved by applying a rigid rotation matrix to (5), such that the geometry pitches at an angle $\delta$ about an axis parallel to the $y$-axis and passing through the $C o G=\left(x_{G}, 0, z_{C o G}+z_{G}\right)$, as:

$\left\{\begin{array}{l}x(\rho, \theta)=f\left(\frac{\rho}{\cos \delta}\right) \cos \theta \cos \delta+\rho \tan \delta-z_{C o G} \sin \delta+x_{G} \\ y(\rho, \theta)=f\left(\frac{\rho}{\cos \delta}\right) \sin \theta \\ z(\rho, \theta)=-f\left(\frac{\rho}{\cos \delta}\right) \cos \theta \sin \delta+\rho+z_{C o G}(1-\cos \delta)+z_{G}\end{array}\right.$

Such an approach is investigated in (Giorgi and Ringwood, 2018a). The main drawback of the representation in the global frame is related to the complexity of the resulting limits of integrations and the unity vectors $\mathbf{e}_{\rho}$ and $\mathbf{e}_{\ominus}$, which adversely affects the computational time of the numerical integration. 
In contrast, following option (b), the formulation of the unity vectors is as simple as in the vertical case. In fact, the geometry is described in the body-fixed frame $(\hat{x}, \hat{y}, \hat{z})$, with the origin at the centre of gravity of the device, by means of the cylindrical coordinates $(\hat{\rho}, \hat{\theta})$ :

$\left\{\begin{array}{l}\hat{x}(\hat{\rho}, \hat{\theta})=f(\hat{\rho}) \cos \hat{\theta} \\ \hat{y}(\hat{\rho}, \hat{\theta})=f(\hat{\rho}) \sin \hat{\theta}, \quad \hat{\theta} \in[-\pi, \pi) \wedge \hat{\rho} \in\left[\hat{\rho}_{1} \hat{\rho}_{2}\right] \\ \hat{z}(\hat{\rho}, \hat{\theta})=\hat{\rho}\end{array}\right.$

However, in option (b), the pressure must be mapped from the global frame into the body-frame. This is simply done by applying a rigid rotation and translation to the body-frame $(\hat{x}, \hat{y}, \hat{z})$, in order to define the inertial frame $(x, y, z)$ as:

$\left\{\begin{array}{l}x=\hat{x} \cos \delta+\hat{z} \sin \delta+x_{G} \\ y=\hat{y} \\ z=-\hat{x} \sin \delta+\hat{z} \cos \delta+z_{G}+z_{C o G}\end{array}\right.$

Option (b) is more advantageous than option (a), since the increase in complexity required to define the unity vectors in the global frame (option (a)) is significantly higher than the increase in complexity due to the pressure mapping into the body-frame (option (b)). Moreover, in the body frame, simplified geometric properties can be exploited; in particular, the surge component of a disc, and the heave component of the lateral surface of a cylinder, are always null in the body frame. Furthermore, if a six-DoF model is considered, the yaw component of any axisymmetric geometry is also always null in the body frame.

Finally, for both options (a) and (b), the FK force integrals must be solved numerically using, for example, a 2D-quadrature scheme (Shampine, 2008). The computation time depends on the integration scheme utilized, and on the relative and absolute tolerances used to approximate the integral, which have been set to 0.1 and 100, respectively, based on the numerical value of the expected forces, and on a sensitivity analysis. Furthermore, it is convenient to compute the static and dynamic FK forces together, in order to reduce the number of integrals to be solved.

The ultimate value of the computation time depends on the complexity of the geometry. Indeed, real WEC buoys may be a combination of different sections, typically cylinders and cones, as in the case shown in Table 1 , with each section of the buoy requiring an individual integral. For the CorPower geometry, which is described in Sect. 3, it is found that the numerical integration scheme (option (b)) is, on average, about 50 times slower than the algebraic integration, where the computational time of the VFK method is of the order of $1 \cdot 10^{-4}$. Note that option (a) is about 80 times slower than the algebraic integration. However, it is important to point out that all calculations are performed in Matlab, which is between one and two orders of magnitude slower than lower level coding languages, such as C or Fortran (Wendt et al., 2017).

It is worth to remark that the computational convenience of the proposed method is the analytical representation of the integral, which is needless of a numerical mesh-based computation of the wetted surface. Indeed, the mesh-based nonlinear FK software LAMSWEC (Gilloteaux, 2007), for example, although coded in Fortran, is about one order of magnitude slower than the method proposed in this paper (Giorgi and Ringwood, 2017a,2017b,2017c; Gilloteaux, 2007).

\section{Validation of modelling approach}

Several checks have been performed, in order to ensure the code, implementing the nonlinear FK force calculations, to be correct and reliable. The surface of several simple geometries, generated with either the vertical or rotated cylindrical coordinates, has been visually inspected, with particular care on the ability to correctly represent the intersection between the rotated buoy and the free surface elevation. All the algebraic calculations have been checked via the symbolic

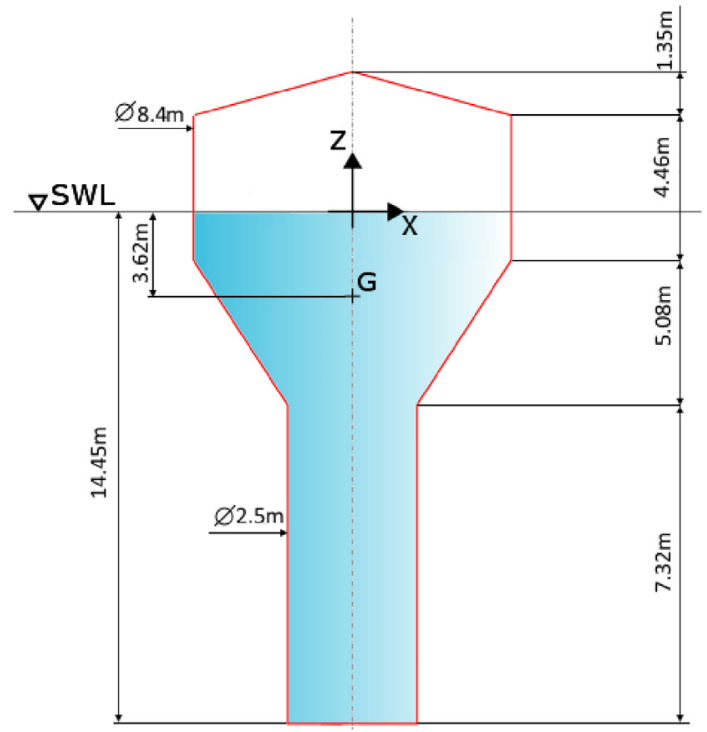

Fig. 2. Shape and dimensions of the case study buoy, inspired by the CorPower device.

calculation software Mathematica (Wolfram, 2017), and effectively compared with numerical integration. Furthermore, surface and volume integrals have been computed, in order to calculate the surface and volume of sample geometries (cylinders and cones), either vertical or rotated.

\subsection{Comparison with WAMIT}

Linear boundary element (BEM) codes can be used to validate the static and dynamic FK integrals; for very small motion and wave amplitudes, the nonlinear models should produce results consistent with BEM codes. Hereafter, a particular device is taken into account for the computation of FK forces, based on the CorPower device geometry (CorPower, 2017), whose shape is shown in Table 1. Based on (Todalshaug et al., 2016), the device dimensions are shown in Fig. 2.

The BEM code WAMIT (Inc, 2013) is used to calculate the linear surge, heave, and pitch hydrostatic stiffness and the linear FK force for the vertical device. The nonlinear static FK forces are calculated with the VFK and RFK method, using very small displacements in heave and pitch ( $0.01 \mathrm{~m}$ and $0.01 \mathrm{rad}$, respectively), while the nonlinear dynamic FK forces are computed with waves of different period $T_{w}$, and height $H_{w}=0.01 \mathrm{~m}$. The three models (LFK, VFK, and RFK) return identical results, validating the method in linear conditions. Note that, in general, the RFK method converges to the VFK method when the pitch angle is zero.

A second configuration is run in WAMIT, considering the geometry statically rotated by $15^{\circ}$ around its centre of gravity, in order to compute the hydrostatic stiffness and FK force linearized around a non-zero pitch angle, and validate the RFK method for a rotated geometry. Fig. 3 shows the validation of the dynamic FK force amplitude coefficients and phase lags, with respect to the free surface elevation. On the one hand, in Fig. 3, the WAMIT calculations relate to a rotated geometry, in order to provide a preliminary validation of the RFK modelling approach, and verify its implementation; on the other hand, Fig. 3 also includes the VFK model, applied to a vertical geometry, in order to discuss some nonlinear effects induced by pitch angles.

Both amplitude and phase of LFK and RFK overlap, validating the modelling approach. Conversely, while phases for the vertical buoy (VFK) are constant (in the considered range of $T_{w}$ ), they are frequencydependent for the rotated one (RFK). Furthermore, while amplitudes of surge and heave are almost identical, significant differences are found for the pitch torque. In fact, for a rotated buoy, both surge and heave 

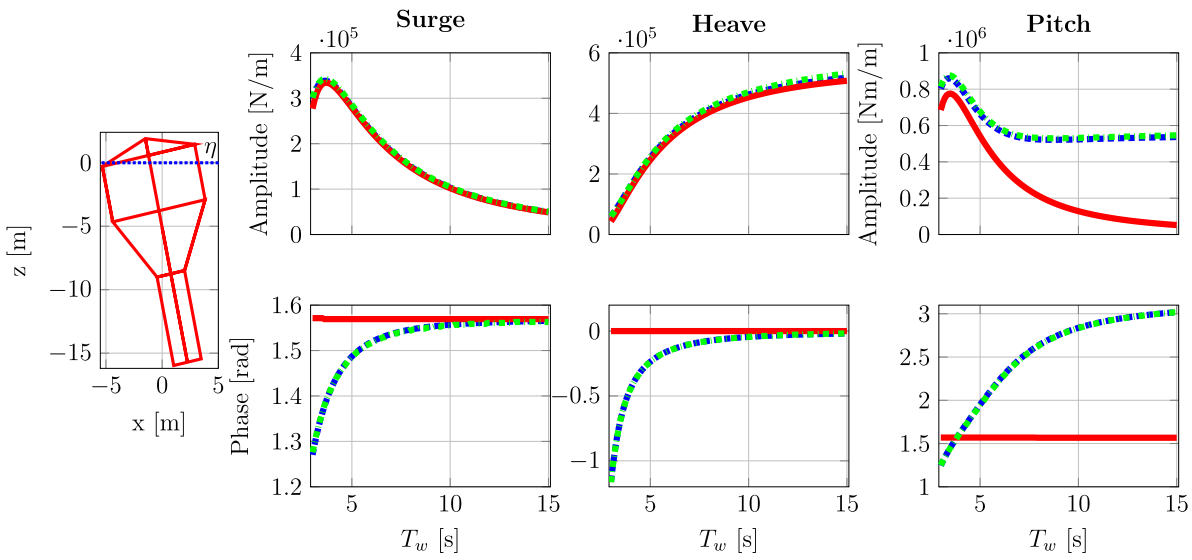

contribute to the pitch torque which, indeed, resembles the profile of the surge curve for short periods (large surge, small heave), and vice versa at high periods (small surge, large heave).

\subsection{Comparison with tank tests}

Comparing force components with WAMIT results, in linear conditions, is a simple way to verify the correctness of the implementation, and to validate the modelling approach. However, conclusions can be drawn only for small relative displacements of the body, with respect to the free surface. A complete validation of the model, particularly with larger waves and displacements, ideally requires wave tank data. The CorPower device was tested in November 2014 in the wave tank at Ecole Centrale de Nantes, France, and results are published in (Todalshaug et al., 2016). Although not in possession of the actual data from the tank, it is possible to reproduce the experimental setup within the mathematical model, and compare results, in order to achieve a preliminary validation.

One of the main objectives of the experimental campaign was to test the effectiveness of the WaveSpring (WS) mechanism, which acts as a negative spring. Considering a linear damper (LD) as a power take-off (PTO), two configurations are tested, namely with (LD + WS) or without (LD) WaveSpring. Refer to (Todalshaug et al., 2016) for full details about the experimental setup, and the mathematical model used for the WaveSpring mechanism. Replicating the same experimental conditions, the motion of the device is simulated, as discussed in Sect. 5, using both the VFK and the RFK model. Results are shown in Fig. 4. From the comparison, it can be concluded that the agreement between

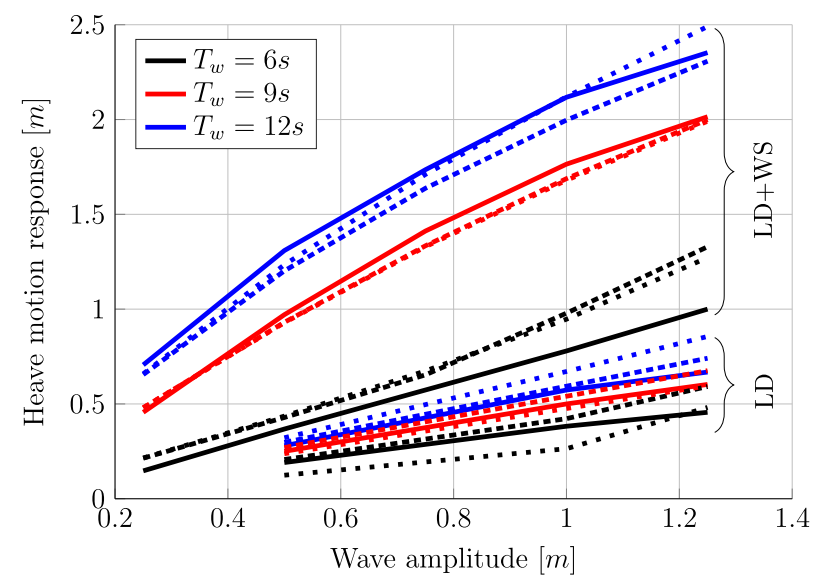

Fig. 4. Comparison of the heave response to regular waves, obtained in wave tank tests in (Todalshaug et al., 2016) (solid line), and numerical simulations, using the VFK model (dotted line) and the RFK model (dashed line).
Fig. 3. Froude-Krylov forces, used for validation, in linear conditions, of the RFK method $(\uplus \cdots)$, compared with linear results for the CorPower buoy rotated by $15^{\circ}(-1)$. For comparison, the VFK method is presented ( $($ ), which refers to a vertical buoy. numerical and experimental results is fairly good, in both configurations. Furthermore, the two nonlinear models (VFK and RFK) perform similarly in predicting the heave motion response, and hence power production, as is further discussed in Sect. 5.

\section{Parametric study}

The relative disparity in accuracy between the three methods (LFK, VFK, and RFK) depends on four different factors, two concerning the position of the device $\left(z_{G}\right.$ and $\delta$ ), and two concerning the wave characteristics $\left(H_{w}\right.$ and $\left.T_{w}\right)$. Indeed, regular waves are considered, in order to study the specific dependence on $H_{w}$ and $T_{w}$. Importantly, higher waves induce larger variations in the wetted surface during the wave cycle, inducing more significant nonlinearities. However, the LFK and VFK methods converge for small values of $z_{G}$; similarly, VFK and RFK converge for small values of $\delta$. Given the typical oscillatory response of a WEC, differences between models vanish in that part of the response cycle corresponding to a zero crossing, while they are maximal at the peaks and troughs of the oscillation, as might be expected.

In order to understand how linear and nonlinear models vary and differ from each other, and how they individually depend on $z_{G}$ and $\delta$, surge, heave, and pitch FK forces are computed, for several different regular waves, with the device fixed in a particular position. The following ranges are considered: $T_{w} \in[4 s, 15 s]$, with $1 s$ step; $H_{w} \in[0.5 m, 3 m]$, with $0.5 m$ step; $z_{G} \in[-3 m, 3 m]$, with $0.25 m$ step; $\delta \in\left[-15^{\circ}, 15^{\circ}\right]$, with $1.5^{\circ}$ step. Only linear waves have been considered, in order to focus the discussion just on nonlinearities related to FK force calculations. In this section, only static FK forces, in the body-frame, are discussed. The effect of dynamic FK forces is taken into account in Sect. 5 , where the full dynamic response to incoming waves is simulated.

The static heave FK force $\left(F_{F K_{s t}}^{h}\right)$ is linear, for the VFK model, when $\eta$ intersects the cylinder section, while becoming nonlinear in the cone section, since the cross sectional area is not constant. A more general representation of how $F_{F K_{s t}}^{h}$ varies can be achieved using an "equivalent" stiffness, for each value of $z_{G}$, where $F_{F K_{S t}}^{h}$ is divided by $z_{G}$. Fig. 5 shows such an equivalent stiffness for a particular wave, at $\delta=15^{\circ}$, and varying $z_{G}$. Results for the nonlinear VFK and RFK models are represented by areas, since $F_{F K_{s t}}^{h}$ is not constant. Indeed, Fig. 5 shows the variability of the static FK force, due to the free surface elevation, within one wave cycle.

The VFK model results coincide with those for the LFK model, for small vertical displacements, since $\eta$ intersects the buoy only at the cylinder section, while differences are found at larger $z_{G}$. Conversely, the RFK model is always nonlinear, since the actual rotated geometry is considered. Note that a larger equivalent stiffness for small $z_{G}$ does not mean a larger error; in fact, the difference appears larger just because $F_{s t}^{h}$ is divided by a smaller $z_{G}$.

The nonlinearity/variability of $F_{F K}^{h}$, for both the VFK and RFK 


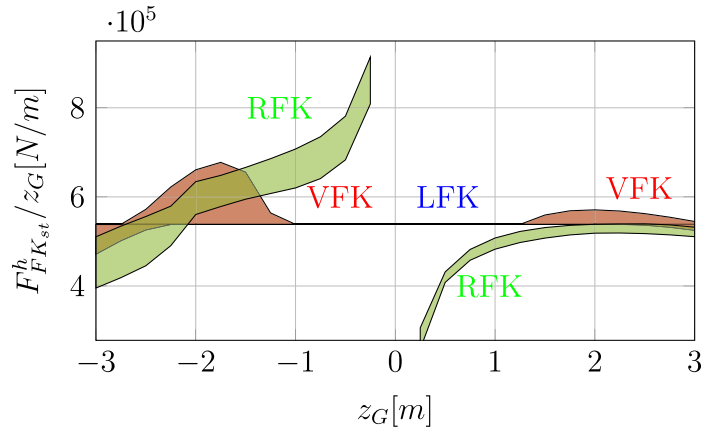

Fig. 5. Equivalent static heave Froude-Krylov force for the CorPower buoy, rotated by $\delta=-15^{\circ}$, subject to a wave with $H_{w}=2 m$ AND $T_{w}=10 \mathrm{~s}$, according to LFK (-), VFK ( $\square$ ), and RFK ( $\square$ ) models.

models, depends on which buoy section is engaged by $\eta$. In particular, larger errors are introduced by the top cone (engaged when $z_{G}$ is large and negative), which has a more pronounced slope than the bottom cone (engaged when $z_{G}$ is large and positive), since the rate of change of the cross sectional area of the top cone is greater. Note that similar graphs can be drawn for different $T_{w}$ and $H_{w}$. While the equivalent heave stiffness is largely insensitive to the wave period, larger wave heights introduce larger errors, since more significant changes in the wetted surface appear.

Finally, the static pitch FK torque $\left(T_{F K_{s t}}^{p}\right)$ is considered. Fig. 6 presents $F_{s t}^{p}$ for different $z_{G}$ and $\delta$, for a sample wave of $T_{w}=10 \mathrm{~s}$ and $H_{w}=2 m$. Only positive pitch angles are shown, since the results are symmetric with respect to the pitch angle.

Given the variability of nonlinear $T_{F K_{s t}}^{p}$, as shown in Fig.??, the mean torque is presented in Fig. 6, along with error bars, whose length is equal to the standard deviation of the static torque, over one wave period. Overall, the variability is quite small, compared to the mean values, apart from a very negative $z_{G}$, where the highly nonlinear top cone intersects $\eta$.

Obviously, the RFK model significantly overlaps with the LFK model for very small $z_{G}$, and diverges from it with when the absolute value of either $\delta$ or $z_{G}$ increases. Furthermore, for vertical displacements larger than $1.75 \mathrm{~m}$, the static torque changes sign, contriving to drive the buoy away from the equilibrium position, as opposed to acting as a restoring torque, which is the case for a linear model. In fact, for $z \geq 1.75 \mathrm{~m}$, the metacentre falls below the centre of gravity, which is the condition for pitching instability (Biran and Pulido, 2013).

Finally, as in Figs. 5 and 6 shows a relative insensitivity of $T_{F K_{S t}}^{p}$ to $T_{w}$, while larger values of $H_{w}$ cause slightly smaller absolute mean values of $T_{F K_{s t}}^{p}$, and slightly increased variability.

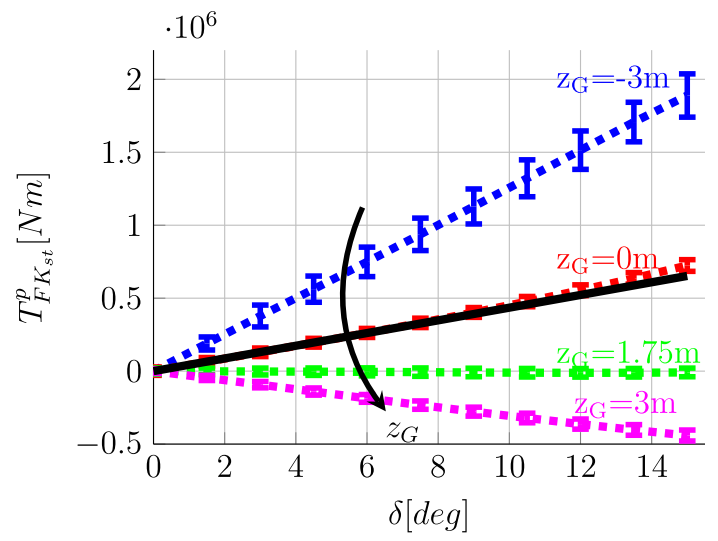

Fig. 6. Static pitch Froude-Krylov force for the CorPower buoy, subject to a wave with $H_{w}=2 m$ and $T_{w}=10 \mathrm{~s}$, according to LFK and VFK (solid line), and RFK (dashed line) models, for different $z_{G}$.
Table 2

Model parameters, in surge, heave, and pitch, based on (Todalshaug et al., 2016).

\begin{tabular}{llll}
\hline & Surge & Heave & Pitch \\
\hline$M$ & $75,130 \mathrm{~kg}$ & $75,130 \mathrm{~kg}$ & $2.36 \mathrm{e} 6 \mathrm{kgm}^{2}$ \\
$K_{\text {moo }}$ & $7.2 \mathrm{e} 4 \mathrm{~N} / \mathrm{m}$ & $2 \mathrm{e} 4 \mathrm{~N} / \mathrm{m}$ & $9.5 \mathrm{e} 5 \mathrm{Nm} / \mathrm{m}$ \\
$A_{d}$ & $64.4 \mathrm{~m}^{2}$ & $55.42 \mathrm{~m}^{2}$ & $64.4 \mathrm{~m}^{2}$ \\
$C_{d}$ & 0.35 & 0.35 & 1 \\
$T_{n}$ & $13 \mathrm{~s}$ & $3 \mathrm{~s}$ & $7.5 \mathrm{~s}$ \\
\hline
\end{tabular}

\section{Complete hydrodynamic model}

The motion of the device is described by Newton's second law, in vectorial form, for 3 degrees of freedom, written in the body-fixed frame of reference:

$M \ddot{\mathbf{x}}=\mathbf{f}_{F K_{s t}}+\mathbf{f}_{F K_{d y}}+\mathbf{f}_{d}+\mathbf{f}_{r a d}+\mathbf{f}_{v i s}+\mathbf{f}_{C o r}+\mathbf{f}_{m o o}+\mathbf{f}_{P T O}$,

where $M$ is the inertial matrix, $\mathbf{x}=(\hat{x}, \hat{z}, \delta)$ is the state vector in the body-fixed frame, $\mathbf{f}=\left(F^{s}, F^{h}, T^{p}\right)$ is the generalized force vector, composed of surge and heave forces, and the pitch torque. The other force components in (12) are $\mathbf{f}_{d}$, the diffraction force, $\mathbf{f}_{r a d}$, the radiation force, $\mathbf{f}_{v i s}$, the viscous force, $\mathbf{f}_{C o r}$, the Coriolis force, $\mathbf{f}_{\text {moo }}$, the mooring force, and $\mathbf{f}_{P T O}$, the PTO force. Note that $\mathbf{f}_{P T O}$ acts on the axis of the device, therefore along $\hat{z}$. The important numerical parameter values used in the model are summarized in Table 2.

The advantage of writing (12) in the body frame, with the origin at the centre of gravity, is that $M$ is diagonal, and constant. However, Coriolis forces must be included, since the frame is accelerating, and is therefore non-inertial, as

$\mathbf{f}_{C o r}=\left(\begin{array}{c}m \dot{\hat{z}} \dot{\delta} \\ m \dot{\hat{x}} \dot{\delta} \\ 0\end{array}\right)$,

where $m$ is the mass of the device. The radiation forces are composed of added mass and a convolution integral (Cummins, 1962), which is substituted by a more computationally convenient state space representation (Perez and Fossen, 2009). Note that surge and pitch are coupled modes, due to $\mathbf{f}_{F K_{s t}}$ and $\mathbf{f}_{r a d}$. Mooring forces are simply modelled as linear springs. The stiffness of the mooring lines $\left(K_{\text {moo }}\right)$ has been chosen in order to match the natural period $\left(T_{n}\right)$ in surge and heave, which is available in the literature (Todalshaug et al., 2016). The mooring stiffness, in pitch, is assumed to be equal to the mooring stiffness in surge multiplied by the distance between the centre of gravity and the mooring point (13.18 m) (Todalshaug et al., 2016). Finally, viscous forces are represented by means of a Morison-like term, in surge and heave (Bhinder et al., 2011), and pitch (Mundon et al., 2017):

$\mathbf{f}_{v i s}=-\frac{1}{2} \rho_{w}\left(\begin{array}{c}A_{d}^{s} C_{d}^{s} \dot{\hat{x}}|\dot{\hat{x}}| \\ A_{d}^{h} C_{d}^{h} \dot{\hat{z}}|\dot{\hat{z}}| \\ A_{d}^{s} R^{3} C_{d}^{p} \dot{\delta}|\dot{\delta}|\end{array}\right)$,

where $\rho_{w}$ is the water density, $A_{d}$ is the cross sectional area of the buoy perpendicular to the flow, $C_{d}$ is the drag coefficient, and $R$ is the radius of the larger cylindrical section of the buoy. The superscripts $(s, h, p)$ of $A_{d}$ and $C_{d}$ refers to surge, heave, and pitch, respectively. Including a viscous drag term is likely to be of particular importance when nonlinear FK forces are considered (Giorgi and Ringwood, 2017c), especially with the eventuality of pitching instability or parametric roll (Babarit et al., 2009). Drag coefficients are experimentally identified, by means of free decay tests (Todalshaug et al., 2016).

The PTO is modelled as a linear damper, therefore employing a simple resistive control. Previous studies show that resistive control does not introduce any significant nonlinearity in the system, for the 


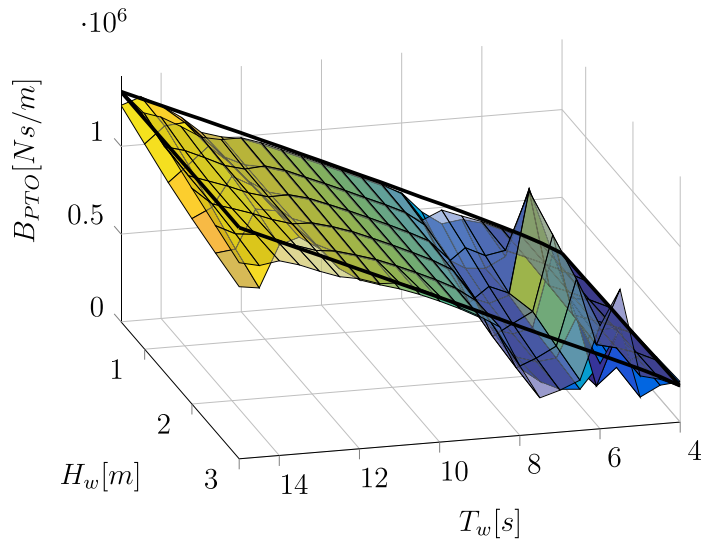

Fig. 7. Optimal PTO coefficient, according to LFK $(\boldsymbol{m})$, VFK (transparent surface), and RFK (opaque surface).

case where the device is purely heaving (Giorgi and Ringwood, 2017c). However, when pitching point absorbers are considered, as in this paper, significant nonlinear effects arise even with a resistive control strategy. Undoubtedly, more aggressive control strategies, like latching control or reactive control, would induce a further degree of nonlinearity in the response of the system.

The PTO coefficient $\left(B_{\text {РTO }}\right)$ is calculated, for each model, and each wave condition, in order to optimize the power output. The resulting PTO coefficients, for the regular wave conditions considered, are presented in Fig. 7.

The PTO coefficients for the LFK model are represented by a solid line, since the surface is mainly flat. In fact, since the only nonlinearity in the LFK model is the viscous drag force (rather small), the optima are relatively insensitive to changes in $H_{w}$, but are linearly increasing with $T_{w}$, in accordance with the theoretical result for maximum power extraction (Falnes, 2002). Conversely, nonlinear effects are evident for the VFK (transparent surface) and RFK (opaque surface) models, with an overall good agreement between the two surfaces. In particular, nonlinearities, represented by the differences between the LFK model and the nonlinear models, are more evident for $T_{w}$ between $5 \mathrm{~s}$ and $8 \mathrm{~s}$ (close to the pitch natural period), and between 13s and 15s (close to the surge natural period, which is coupled with pitch). Otherwise, dominantly linear behaviour is found, in particular at low $H_{w}$, with the three surfaces significantly overlapping. It is therefore reasonable to infer that nonlinearities become important when the pitch mode is sufficiently excited. In fact, Fig. 8 shows the maximum pitch angle excursions, for the LFK and RFK models (the intermediate nonlinear VFK model is not represented for clarity).

Larger pithing motions are evident from the nonlinear RFK model, with a maximum angle of $36.8^{\circ}$, as opposed to $22.2^{\circ}$ for the LFK model. Such a result is consistent with the pitching restoring force variations, with the eventuality of pitching instability, as discussed in Sect. 4, and shown in Fig. 6. Such an instability, due to nonlinear effects, is

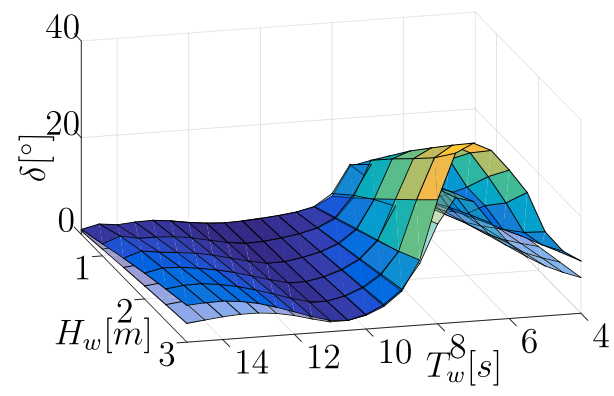

Fig. 8. Maximum pitching angle excursion, according to LFK (transparent surface), and RFK (opaque surface).

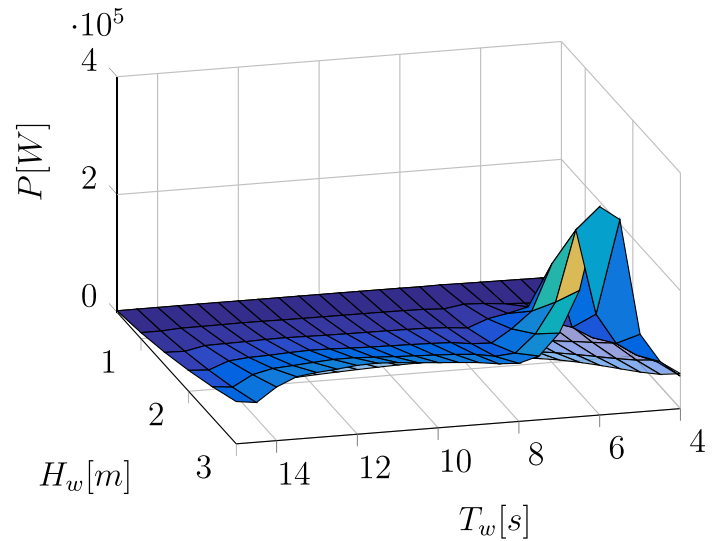

Fig. 9. Maximum absorbed power, according to LFK (transparent surface), and RFK (opaque surface).

ultimately beneficial to the power extraction, as shown in Fig. 9. In fact, in the range of $T_{w}$ where $\delta$ is larger, and for larger $H_{w}$, a significantly higher power is calculated by the RFK model, compared to the LFK one. Otherwise, the LFK and RFK model power results significantly overlap. The power surface for the VFK model (not shown for clarity) is generally similar to the RFK curve, consistently with the trend shown in Fig. 7.

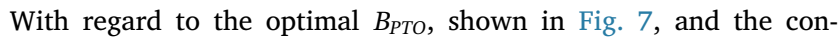
sequent optimal power, shown in Fig. 9, it is important to highlight the fact that such results are optimal relative to the specific model used. For example, the optimal PTO coefficients for the LFK model, are suboptimal when applied to the real device, which is the real target of the optimization. Therefore, the power output of the real device depends on the fidelity of the model used, and on the sensitivity of the power to $B_{P T O}$. In fact, if the power has a low sensitivity to variations in the optimal $B_{P T O}$, it may be acceptable to have low accuracy in the model used for the optimization, so that large errors in $B_{P T O}$ will cause small power losses. Conversely, if the sensitivity to $B_{\text {РтО }}$ is high, the optimization model is required to be accurate, in order to avoid large power losses.

A measure of the power sensitivity to $B_{\text {PTO }}$ can be built as the ratio between the suboptimal and the optimal power. Assuming that the RFK model is the most accurate, the optimal PTO coefficient for the LFK and VFK models are applied to the RFK model, hence producing suboptimal power outputs. Clearly, optimal PTO coefficients, computed with the RFK model, produce optimal power outputs. Fig. 10 shows the power ratios for a representative wave height of $2 \mathrm{~m}$.

As expected, there is some power loss (ratio lower than unity) only for $T_{w}$ within the range between $5 \mathrm{~s}$ and $8 \mathrm{~s}$, and between $13 \mathrm{~s}$ and 15s. In fact, elsewhere, the optimal parameters of the three models are

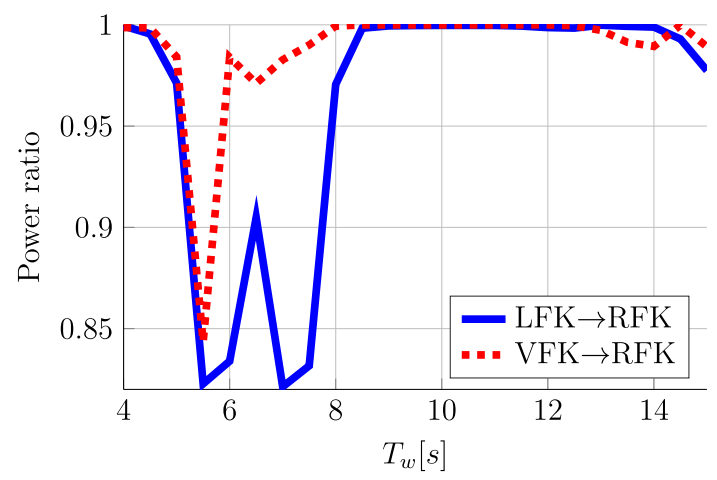

Fig. 10. Measure of sensitivity of the power output to the PTO coefficient, defined as the suboptimal power normalized by the optimal power, with $H_{w}=2 m$. The suboptimal power is computed using the optimal PTO coefficients, according to either the LFK or the VFK model, in the RFK model. 
Table 3

Relative computational time, defined as the ration between the simulation time and the computational time.

\begin{tabular}{ll}
\hline & $t_{\text {rel }}$ \\
\hline LFK & $3 \cdot 10^{-4}$ \\
VFK & $8 \cdot 10^{-3}$ \\
RFK & $4 \cdot 10^{-1}$ \\
\hline
\end{tabular}

essentially the same, as shown in Fig. 7. Furthermore, in the nonlinear region, where the differences are more evident, it is found that the power loss using the VFK $B_{P T O}$ is overall much smaller: a part from $T_{w}=5.5 \mathrm{~s}$, the power loss with the VFK optima is smaller than $3 \%$, while the power loss using the LFK optima reaches a peak of $18 \%$. Similar trends are found for other wave heights, with larger ratios at larger $H_{w}$, due to larger nonlinear effects at more energetic wave conditions.

Therefore, based on Fig. 10, it can be concluded that one favourable option would be to use the VFK model for the control optimization loop, rather than the LFK model. On the other hand, based on Fig. 9, it is convenient to perform the power absorption assessment of the device using the RFK model. Indeed, the optimization loop has a more stringent requirement on the computational time, which is better met by the VFK model than the RFK one. In contrast, the power production assessment requires higher accuracy and accepts longer computational times, since simulations are run once, instead of iteratively.

An average measure of relative computational time $\left(t_{r e l}\right)$ of the three models is presented in Table 3 , defined as the ratio between the simulation time and the run time. Such a ratio shows that all models are able to compute in real time, since the ratio is lower than unity in all cases. The RFK model computes, on average, 50 times slower than the VFK model which, in turn, is about 25 times slower than the LFK model.

Finally, it is interesting to discuss some further nonlinear effects, captured by the VFK and RFK models, while ignored by the LFK model. A representative indicator of nonlinear behaviour of a system is the frequency content of its response to an external excitation. In particular, when a monochromatic input is considered, linear systems respond at one and same frequency of the exciting input. Conversely, appropriately excited nonlinear systems may transfer some energy from the exciting frequency to its multiples or, in some cases, fractions, generating superharmonics and sub-harmonics, respectively. In particular, the response of systems with nonlinear restoring forces typically shows super- and/or sub-harmonics components (Hayashi, 1953).

With respect to the pitch response, shown in Fig. 8, for wave conditions at small pitch angles, the response of the three models is predominantly linear. When the pitch angle increases, super-harmonics are manifest (for the VFK and RFK models), as shown in Fig. 11, where $\omega_{\text {ratio }}$ is the ratio between the wave frequency and the frequency components of the fast-Fourier-transform (FFT) of the response. Furthermore, nonzero mean values are present (at $\omega_{\text {ratio }}=0$ ), which are due to a non-zero mean nonlinear FK force, acting on the system as drift force.

Note that, as far as harmonic generation is concerned, there is good agreement between the VFK and RFK models. Super-harmonics and

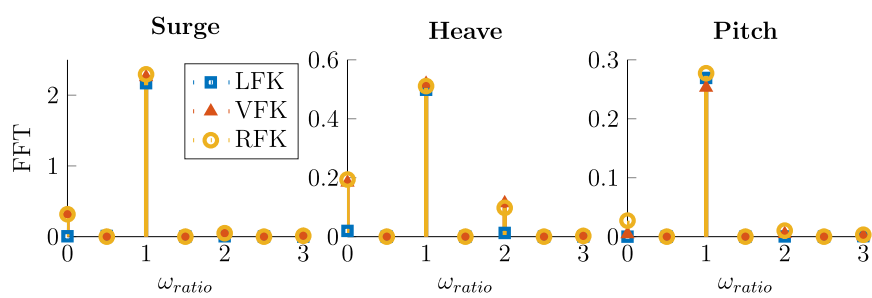

Fig. 11. Fast-Fourier-transform (FFT) of the response of the device, subject to an incoming wave of $T_{w}=8 \mathrm{~s}$ and $H_{w}=2 \mathrm{~m}$, where $\omega_{\text {ratio }}$ is the ratio between the wave frequency and the frequency components of the response.

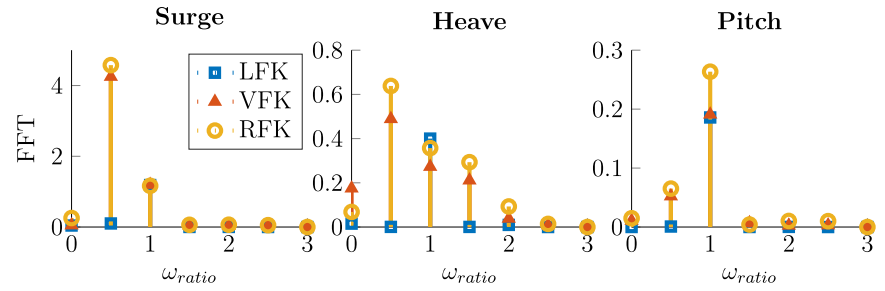

Fig. 12. Fast-Fourier-transform (FFT) of the response of the device, subject to an incoming wave of $T_{w}=6 \mathrm{~s}$ and $H_{w}=2 \mathrm{~m}$, where $\omega_{\text {ratio }}$ is the ratio between the wave frequency and the frequency components of the response.

mean-offsets are quite common nonlinear behaviours, which are typically induced by powers of the input. For the wave condition of Fig. 11, as well as in many other wave conditions, for $T_{w}$ between 5 s and $9 \mathrm{~s}$, and greater than $12 \mathrm{~s}$, a significant amount of spectral energy is found at $\omega_{\text {ratio }}$ of 0,1 , and 2 .

However, for some wave conditions, in particular at $T_{w}=6 \mathrm{~s}$, and $H_{w}$ from $1.5 \mathrm{~m}$ to $3 \mathrm{~m}$, sub-harmonics are found as well $\left(\omega_{\text {ratio }}=0.5\right)$, of which an example is shown in Fig. 12. Fractional components are usually generated beyond an amplitude threshold of $\eta$ (Eller, 1973), explaining why they are visible only for $H_{w}$ greater than $1.5 \mathrm{~m}$. Furthermore, sub-harmonics appear only in multi-DoF nonlinear systems (Eller, 1973), where sub-harmonics generated when the excitation frequency is twice the natural frequency of one of the Dofs (Eller, 1973). Since, at $T_{w}=6 \mathrm{~s}$, the excitation frequency is about twice the surge natural frequency, it can be inferred that the sub-harmonics are caused by a nonlinear excitation of the surge degree of freedom.

\section{Conclusions}

This paper discusses the importance and relevance of nonlinear FroudeKrylov force representation for axisymmetric wave energy converters, moving in surge, heave, and pitch. Though limiting (but not excessively so), the assumption of an axisymmetric device makes the analytical description of the surface of the device possible, therefore avoiding the use of time-consuming mesh-based approaches. In particular, two computationally convenient methods are proposed: one using a numerical integration scheme, the other algebraically solving the Froude-Krylov integrals, and relying on the assumption of small pitch angles.

As a case study, the CorPower wave energy device is considered. A parametric study reveals significant nonlinearities in surge and heave appear when the fluid intersects the conical sections of the buoy, a condition depending on the heave position $z_{G}$, and the wave height $H_{w}$. In contrast, nonlinearities in pitch appear to be important for a wide range of device positions $\left(z_{G}\right.$ and $\delta$ ) and waves $\left(T_{w}\right.$ and $\left.H_{w}\right)$.

For the response of the WEC to incoming regular waves, nonlinearities are particularly evident for larger pitch angles, where the optimal control parameters, estimated with the linear model, are significantly different from those of the numerical-nonlinear model, while the algebraic-nonlinear model shows, overall, a good agreement. Consequently, the sensitivity of the power absorbed to inaccuracies on the control parameters is studied, highlighting that the algebraic-nonlinear model would be a suitable model for a control optimization strategy, due to its computational convenience and overall accuracy. On the other hand, the numerical integration approach is able to highlight important nonlinear effects, like pitching instability, and the creation of sub- and super-harmonics. Therefore, the numerical integration approach would be more appropriate for simulation and maximum load estimation.

\section{Acknowledgment}

This paper is based upon work supported by Science Foundation Ireland under Grant No. 13/IA/1886. 


\section{Appendix A. Algebraic solution}

For a vertical axisymmetric geometry, the Froude-Krylov integrand, shown for example in (6), is composed of the pressure, shown in (1), and the unity vectors, shown in (7) and (8).

Algebraic solutions are available under the assumption of infinite water depth conditions, and expanding the cosine term of the pressure. Furthermore, Wheeler stretching is applied. Therefore, the following pressure, expressed in cylindrical coordinates, is used in the integrals:

$p=-\gamma z+\gamma a \mathrm{e}^{\chi_{s}(z-\bar{\eta})}$

$\left(\cos \omega t+\chi f(\rho) \cos \theta \sin \omega t-\frac{(\chi f(\rho) \cos \theta)^{2}}{2} \cos \omega t-\frac{(\chi f(\rho) \cos \theta)^{3}}{6} \sin \omega t+\frac{(\chi f(\rho) \cos \theta)^{4}}{24} \cos \omega t+\frac{(\chi f(\rho) \cos \theta)^{5}}{120} \sin \omega t\right)$

where $\chi_{s}=\frac{\chi h}{\bar{\eta}+h}$ is the stretched wave number. Although the McLaurin expansion, up to the fifth order, is composed of six terms, three of them always disappear, since odd powers of $\cos \theta$ integrate to zero, when $\theta \in[-\pi, \pi$ ). Clearly, which terms cancel out from (A.1) depends on the degree of freedom of the specific unity vectors considered, which may contain either $\cos \theta \operatorname{or} \sin \theta$.

The solution of the nonlinear FK integrals for a circle, cylinder, and cone with vertical axis are hereafter presented. For any axisymmetric vertical device, the static surge and pitch FK forces are zero, because the integrand contain a $\cos \theta$.

\section{Appendix A.1. Cylinder}

For the lateral surface of a vertical cylinder, characterized by $f(\rho)=R$, the following results are obtained

$\mathbf{e}_{\rho} \times \mathbf{e}_{\theta}=R\left(\begin{array}{c}-\cos \theta \\ -\sin \theta \\ 0\end{array}\right)$

$<\mathbf{r} \times\left(\mathbf{e}_{\rho} \times \mathbf{e}_{\theta}\right), \mathbf{j}>=-R \rho \cos \theta$

$F_{F K_{s t}}^{h}=0$

$F_{F K_{d y}}^{s}=\frac{\gamma \pi R^{2} \chi}{\chi_{s}} a \sin \omega t\left(\frac{\chi^{2} R^{2}}{8}+\frac{\chi^{4} R^{4}}{192}-1\right)\left[\mathrm{e}^{\chi_{s}(\rho-\bar{\eta})}\right]_{\rho_{1}}^{\rho_{2}}$

$F_{F K_{d y}}^{h}=0$

$T_{F K_{d y}}^{p}=\frac{\gamma \pi R^{2} \chi}{\chi_{s}^{2}} a \sin \omega t\left(\frac{\chi^{2} R^{2}}{8}+\frac{\chi^{4} R^{4}}{192}-1\right)\left[\left(\chi_{s} \rho-1\right) \mathrm{e}^{\chi_{s}(\rho-\bar{\eta})}\right]_{\rho_{1}}^{\rho_{2}}$

\section{Appendix A.2. Disc}

In order to completely describe a cylinder, the bottom disc, which "closes" the cylinder, must be described. For this purpose, polar coordinates are used, as opposed to cylindrical coordinates. The following results are obtained:

$\left\{\begin{array}{l}x(r, \theta)=r \cos \theta \\ y(r, \theta)=r \sin \theta, \quad \theta \in[-\pi, \pi) \wedge r \in[0, R] \\ z(r, \theta)=h_{0}\end{array}\right.$

$\mathbf{e}_{r} \times \mathbf{e}_{\theta}=\left(\begin{array}{c}\cos \theta \\ \sin \theta \\ 0\end{array}\right) \times\left(\begin{array}{c}-r \sin \theta \\ r \cos \theta \\ 0\end{array}\right)=r\left(\begin{array}{l}0 \\ 0 \\ 1\end{array}\right)$

$<\mathbf{r} \times\left(\mathbf{e}_{r} \times \mathbf{e}_{\theta}\right), \mathbf{j}>=-r^{2} \rho \cos \theta$

$F_{F K_{S t}}^{h}=-\gamma \pi R^{2} h_{0}$

$F_{F K_{d y}}^{S}=0$

$F_{F K_{d y}}^{h}=\gamma \pi R^{2} \mathrm{e}^{\chi_{s}\left(h_{0}-\bar{\eta}\right)} a \cos \omega t\left(1-\frac{\chi^{2} R^{2}}{8}+\frac{\chi^{4} R^{4}}{192}\right)$

$T_{F K_{d y}}^{p}=\frac{1}{4} \gamma \pi R^{4} \chi \mathrm{e}^{\chi_{s}\left(h_{0}-\bar{\eta}\right)} a \sin \omega t\left(\frac{\chi^{4} R^{4}}{384}+\frac{\chi^{2} R^{2}}{12}-1\right)$

Where $h_{0}$ is the draft of the disc.

\section{Appendix A.3. Cone}

Finally, for a vertical cone, whose profile of revolution is defined as $f(\rho)=m \rho+q$, the following results stand: 
$\mathbf{e}_{\rho} \times \mathbf{e}_{\theta}=(m \rho+q)\left(\begin{array}{c}-\cos (\theta) \\ -\sin (\theta) \\ m\end{array}\right)$

$<\mathbf{r} \times\left(\mathbf{e}_{\rho} \times \mathbf{e}_{\theta}\right), \mathbf{j}>=-(m \rho+q)(\rho+m(m \rho+q)) \cos \theta$

$F_{F K_{s t}}^{h}=-2 \gamma \pi m\left[m \frac{\rho^{3}}{3}+q \frac{\rho^{2}}{2}\right]_{\rho_{1}}^{\rho_{2}}$

$F_{F K_{d y}}^{s}=-\gamma \pi \chi a \sin \omega t\left[A_{s}(\rho)-B_{s}(\rho)\right]_{\rho_{1}}^{\rho_{2}}$

$F_{F K_{d y}}^{h}=\gamma \pi m a \cos \omega t\left[2 A_{h}(\rho)-\frac{\chi^{2}}{2} B_{h}(\rho)+\frac{\chi^{4}}{32} C_{h}(\rho)\right]_{\rho_{1}}^{\rho_{2}}$

$T_{F K_{d y}}^{p}=-\gamma \pi \chi a \sin \omega t\left[A_{p}(\rho)-\frac{\chi^{2}}{8} B_{p}(\rho)\right]_{\rho_{1}}^{\rho_{2}}$

where

$$
\begin{aligned}
& A_{s}(\rho)=\frac{\mathrm{e} \chi_{s}(\rho-\bar{\eta})}{\chi_{s}^{3}}\left(m^{2}\left(\chi_{s}^{2} \rho^{2}-2 \chi_{s} \rho+2\right)+2 m q \chi_{s}\left(\chi_{s} \rho-1\right)+q^{2} \chi_{s}^{2}\right) \\
& B_{s}(\rho)=\frac{\chi^{2} \chi_{s}(\rho-\bar{\eta})}{8 \chi_{s}^{5}}\left(m^{4}\left(\chi_{s}^{4} \rho^{4}-4 \chi_{s}^{3} \rho^{3}+12 \chi_{s}^{2} \rho^{2}-24 \chi_{s} \rho+24\right)+\right. \\
& \left.4 m^{3} q \chi_{s}\left(\chi_{s}^{3} \rho^{3}-3 \chi_{s}^{2} \rho^{2}+6 \chi_{s} \rho-6\right)+6 m^{2} q^{2} \chi_{s}^{2}\left(\chi_{s}^{2} \rho^{2}-2 \chi_{s} \rho+2\right)+4 m q^{3} \chi_{s}^{3}\left(\chi_{s} \rho-1\right)+q^{4} \chi_{s}^{4}\right) \\
& A_{h}(\rho)=\frac{\mathrm{e}^{\chi_{s}(\rho-\bar{\eta})}}{\chi_{s}^{2}}\left(m\left(\chi_{s} \rho-1\right)+\chi_{s} q\right) \\
& B_{h}(\rho)=\frac{\mathrm{e} \chi_{s}(\rho-\bar{\eta})}{\chi_{s}^{4}}\left(\chi_{s}^{3} q^{3}+3 \chi_{s} m^{2} q\left(\chi_{s}^{2} \rho^{2}-2 \chi_{s} \rho+2\right)+3 \chi_{s}^{2} m q^{2}\left(\chi_{s} \rho-1\right)+m^{3}\left(\chi_{s}^{3} \rho^{3}-3 \chi_{s}^{2} \rho^{2}+6 \chi_{s} \rho-6\right)\right) \\
& C_{h}(\rho)=\frac{\mathrm{e}_{s}(\rho-\bar{\eta})}{\chi_{s}^{6}}\left(\chi_{s}^{5} q^{5}+5 \chi_{s}^{4} m q^{4}\left(\chi_{s} \rho-1\right)+10 \chi_{s}^{2} m^{3} q^{2}\left(\chi_{s}^{3} \rho^{3}-3 \chi_{s}^{2} \rho^{2}+6 \chi_{s} \rho-6\right)+\right. \\
& 10 \chi_{s}^{3} m^{2} q^{3}\left(\chi_{s}^{2} \rho^{2}-2 \chi_{s} \rho+2\right)+5 \chi_{s} m^{4} q\left(\chi_{s}^{4} \rho^{4}-4 \chi_{s}^{3} \rho^{3}+12 \chi_{s}^{2} \rho^{2}-24 \chi_{s} \rho+24\right)+ \\
& \left.m^{5}\left(\chi_{s}^{5} \rho^{5}-5 \chi_{s}^{4} \rho^{4}+20 \chi_{s}^{3} \rho^{3}-60 \chi_{s}^{2} \rho^{2}+120 \chi_{s} \rho-120\right)\right) \\
& A_{p}(\rho)=\frac{\mathrm{e}_{s}(\rho-\bar{\eta})}{\chi_{s}^{4}}\left(3 \chi_{s} m^{3} q\left(\chi_{s}^{2} \rho^{2}-2 \chi_{s} \rho+2\right)+\chi_{s} m q\left(\chi_{s}^{2}\left(2 \rho^{2}+q^{2}\right)-4 \chi_{s} \rho+4\right)+\chi_{s}^{2} q^{2}\left(\chi_{s} \rho-1\right)+\right. \\
& \left.m^{4}\left(\chi_{s}^{3} \rho^{3}-3 \chi_{s}^{2} \rho^{2}+6 \chi_{s} \rho-6\right)+m^{2}\left(\chi_{s}^{3}\left(\rho^{3}+3 \rho q^{2}\right)-3 \chi_{s}^{2}\left(\rho^{2}+q^{2}\right)+6 \chi_{s} \rho-6\right)\right) \\
& B_{p}(\rho)=\frac{\mathrm{e} \chi_{s}(\rho-\bar{\eta})}{\chi_{s}^{6}}\left(\chi_{s}^{4} q^{4}\left(\chi_{s} \rho-1\right)+\chi_{s}^{2} m^{2} q^{2}\left(\chi_{s}^{3}\left(6 \rho^{3}+5 \rho q^{2}\right)-\chi_{s}^{2}\left(18 \rho^{2}+5 q^{2}\right)+36 \chi_{s} \rho-36\right)+\right. \\
& \chi_{s}^{3} m q^{3}\left(\chi_{s}^{2}\left(4 \rho^{2}+q^{2}\right)-8 \chi_{s} \rho+8\right)+5 \chi_{s} m^{5} q\left(\chi_{s}^{4} \rho^{4}-4 \chi_{s}^{3} \rho^{3}+12 \chi_{s}^{2} \rho^{2}-24 \chi_{s} \rho+24\right)+ \\
& 2 \chi_{s} m^{3} q\left(\chi_{s}^{4}\left(2 \rho^{4}+5 \rho^{2} q^{2}\right)-2 \chi_{s}^{3}\left(4 \rho^{3}+5 \rho q^{2}\right)+2 \chi_{s}^{2}\left(12 \rho^{2}+5 q^{2}\right)-48 \chi_{s}+\rho+48\right)+ \\
& m^{4}\left(\chi_{s}^{5}\left(\rho^{5}+10 \rho^{3} q^{2}\right)-5 \chi_{s}^{4}\left(\rho^{4}+6 \rho^{2} q^{2}\right)+20 \chi_{s}^{3}\left(\rho^{3}+3 \rho q^{2}\right)-60 \chi_{s}^{2}\left(\rho^{2}+q^{2}\right)+120 \chi_{s} \rho-120\right)+ \\
& \left.\left.m_{s}^{2} \rho^{2}+120 \chi_{s} \rho-120\right)\right)
\end{aligned}
$$

\section{References}

Babarit, A., Mouslim, H., Clément, A., Laporte-Weywada, P., 2009. On the numerical modelling of the non linear behaviour of a wave energy converter. In: ASME 2009 28th International Conference on Ocean, Offshore and Arctic Engineering. American Society of Mechanical Engineers, pp. 1045-1053.

Bandyk, P., 2009. A Body-exact Strip Theory Approach to Ship Motion Computations. Ph.D. thesis. Michigan.

Bhinder, M.A., Babarit, A., Gentaz, L., Ferrant, P., 2011. Assessment of viscous damping via 3d-cfd modelling of a floating wave energy device. In: Proceedings of the 9th European Wave and Tidal Energy Conference, Southampton, UK.

Biran, A., Pulido, R.L., 2013. Ship Hydrostatics and Stability. Butterworth-Heinemann. CorPower, 2017. Corpower ocean ab. available at. http://www.corpowerocean.com/.

Cummins, W., 1962. The impulse response function and ship motion. Schiffstechnik (9), 101-109.

Eller, A.I., 1973. Fractional-harmonic frequency pairs in nonlinear systems. J. Acoust. Soc. Am. 53 (3), 758-765.

Falnes, J., 2002. Ocean Waves and Oscillating Systems. Cambridge University Press.

Gilloteaux, J.-C., 2007. Mouvements de grande amplitude d'un corps flottant en fluide parfait. application à la récupération de l'énergie des vagues. Ph.D. thesis. Ecole Centrale de Nantes-ECN.
Giorgi, G., Ringwood, J.V., 2017a. Comparing nonlinear hydrodynamic forces in heaving point absorbers and oscillating wave surge converters. J. Ocean Eng. Marine Energy $1-11$.

Giorgi, G., Ringwood, J.V., 2017b. Computationally efficient nonlinear froude-krylov force calculations for heaving axisymmetric wave energy point absorbers. J. Ocean Eng. Marine Energy 3 (1), 21-33.

Giorgi, G., Ringwood, J.V., 2017c. Froude-krylov and viscous drag representations in nonlinear wave energy devices models in the computation/fidelity continuum. Ocean Eng. 141, 164-175.

Giorgi, G., Ringwood, J.V., 2018a. Analytical formulation of nonlinear froude-krylov forces for surging-heaving-pitching point absorbers. In: ASME 2018 37th International Conference on Ocean, Offshore and Arctic Engineering.

Giorgi, G., Ringwood, J.V., 2018b. Relevance of pressure field accuracy for nonlinear froude-krylov force calculations for wave energy devices. J. Ocean Eng. Marine Energy 4 (1), 57-71.

Hayashi, C., 1953. Subharmonic oscillations in nonlinear systems. J. Appl. Phys. 24 (5), 521-529.

Inc, W., 2013. WAMIT v7.0 Manual.

Matusiak, J., 2011Matusiak. On the non-lnon-linear of ship's restoring and the froudekrylov wave load part. Int. J. Naval Architect. Ocean Eng. 3 (1), 111-115.

Mundon, T.R., Rosenberg, B.J., van Rij, J., 2017. Reaction body hydrodynamics for a multi-dof point-absorbing wec. In: 12th European Wave and Tidal Energy Conference 
(EWTEC), Cork.

Penalba, M., Giorgi, G., Ringwood, J.V., 2017. Mathematical modelling of wave energy converters: a review of nonlinear approaches. Renew. Sustain. Energy Rev. 78, 1188-1207. https://doi.org/10.1016/j.rser.2016.11.137. http://www.eeng.nuim. ie/coer/wp-content/uploads/2017/05/J270MRGG-3.pdf.

Perez, T., Fossen, T.I., 2009Perez and Fossen. A Matlab toolbox for paramteric identification of radiation-force models of ships and offshore structures, Modelling. Identification and Control 30 (1), 1-15.

Shampine, L., 2008. Matlab program for quadrature in 2d. Appl. Math. Comp. 202 (1), 266-274.

Tarrant, K.R., March 2015Tarrant. Numerical Modelling of Paramtetric Resonace of a Heaving Point Absorber Wave Energy Converter. Ph.D. thesis. Department of
Mechanical \& Manufacturing Engineering, Trinity College.

Todalshaug, J.H., Asgeirsson, G.S., Hjálmarsson, E., Maillet, J., Möller, P., Pires, P., Guérinel, M., Lopes, M., 2016. Tank testing of an inherently phase-controlled wave energy converter. Int. J. Marine Energy 15, 68-84.

Wendt, F.F., Yu, Y.-H., Nielsen, K., Ruehl, K., Bunnik, T., Touzon, I., Nam, B.W., Kim, J.S., Kim, K.-H., Janson, C.E., et al., 2017. International energy agency ocean energy systems task 10 wave energy converter modeling verification and validation. In: 12th European Wave and Tidal Energy ConferencEuropean Wave and Tidal Energy Conference, Technical Committee of the European Wave and Tidal Energy Conference.

Wolfram, 2017. Wolfram mathematica. available at. http://www.wolfram.com/ matavailable. 